\title{
Nanosecond miniature transmitters for pulsed optical radars
}

\author{
A.V. Filimonov ${ }^{\text {, }}$ V.E. Zemlyakov ${ }^{2}$, V.I. Egorkin ${ }^{2}$, A.V. Maslevtsov ${ }^{I}$, M. C. Wurz ${ }^{3}$ and
}

S.N. Vainshtein ${ }^{4}$

\author{
${ }^{1}$ Peter the Great St. Petersburg Polytechnic University, 195251, Polytechnicheskaya 29, St.- \\ Petersburg, Russia \\ filimonov@rphf.spbstu.ru,avm@spbstu.ru \\ ${ }^{2}$ National Research University of Electronic Technology - MIET, Bld. 1, Shokin Square, \\ Zelenograd, Moscow, Russia, 124498 \\ vzml@rambler.ru, egorkin@qdn.miee.ru \\ ${ }^{3}$ Institute for Microproduction Technology, Leibniz University of Hanover, Garbsen, Germany \\ wurz@impt.uni-hannover.de \\ ${ }^{4}$ Electronics Laboratory, Department of Electrical Engineering, University of Oulu, P.O. Box \\ 4500, FIN-90014 Oulu, Finland University of Oulu \\ vais@ee.oulu.fi
}

\begin{abstract}
The-state-of-the-art in long-distance near-infrared optical radars is utilization the laser-diode-based miniature pulsed transmitters producing optical pulses of 3-10 ns in duration and peak power typically below 40W. The bandwidth of the receiving channel nowadays exceeds $300 \mathrm{MHz}$, and thus the duration of the optical pulses exceeding $3 \mathrm{~ns}$ is a bottleneck in the task of high practical importance, namely increase in the radar ranging precision. Nowadays the speed of the high-current drivers is limited by the speed of a semiconductor switch that is typically field-effect transistor or an avalanche switch. The last one provides faster switching, and development of new avalanche switches is very challenging and important task, but this is not the only factor limiting the transmitter speed. Here we show that not only the switch, but also parasitic inductance in the miniature assembly and type of the capacitor play very important role in solving the problem of long-distance decimeterprecision radar.
\end{abstract}

Keywords. Optical radars, high-speed switching, avalanche drivers, miniature assembly, peak power.

\section{Introduction}

There is a need to generate current pulses of a few nanoseconds in length with an amplitude of $\sim 10-10^{2} \mathrm{~A}$ across a low-ohmic load for a number of commercial applications. This concerns particularly the pumping of high-power broad-stripe laser diodes for pulsed LIDARS and other systems [1, 2, 3]. Large variety of commercial generators providing nanosecond and sub-nanosecond current pulses approaching and even exceeding 100A are available (see, e.g., http://www.fidtechnology.com/), but large size and prices make their application problematic. Much cheaper and compact is making use of avalanche transistor-based Marx circuits [4] providing reliable 
operation after simple modifications [5] in nanosecond and even picosecond switching at dozens amperes [6], or several hundred amperes for a pulses approaching a dozen nanoseconds. In all those examples, however, the transmitter cannot be considered as low cost or miniature (comparable in price and size with encapsulated laser diode), which are the criteria of principal importance for most of applications. The state-of-the-art competing solutions satisfying those criteria are pulsed switches based on latest developments of GaN field-effect transistors (FET), or properly optimized avalanche transistors. Despite GaN FETs can operate at higher repetition rate $(>100 \mathrm{kHz})$, they obviously lose to avalanche transistors in switching speed, when nanosecond and sub-nanosecond high-current switching is required together with the transmitter miniaturization. Thus simplest, cheapest and most effective ways is to make use of high-voltage $(\sim 150-300 \mathrm{~V})$ avalanche transistors $[7,8]$, which is the state-of-the art in optical radars (lidars) utilizing 3-10 ns in duration optical pulses from the laser diodes in 10-100W power range. This duration of the optical pulse becomes a bottleneck of the lidars aiming at longest possible ranging (preferably several kilometres) with high (around a decimetre) ranging precision. Indeed, the bandwidth of the receiving channels based on avalanche detectors exceeds nowadays $300 \mathrm{MHz}$, and thus even $1 \mathrm{~ns}$ in duration optical pulses can be easily detected without noticeable reduction in the detector sensitivity and critical growth in the noise level. Thus development of a laser diode transmitter emitting optical pulses around $1 \mathrm{~ns}$ in duration with as high as possible (at least several dozen W) peak power is the challenging task for long-ranging high-precision radars, and the main problem consists in high-current nanosecond drivers. It is additionally worth noting that a reduction in optical pulse duration well below $1 \mathrm{~ns}$ is possible when pumping current pulse of the laser diode becomes comparable with lasing delay, and various gain-and Q-switching picosecond modes $[9,10,11]$ can be in principle realized, and even submicron precision of the distance measurement was demonstrated for the distance of $700 \mathrm{~m}$ using femtosecond laser [12]. Those modes are used in laboratory practice or for low-power laser transmitters, but they are not very reliable when broad laser diode chips with intrinsic structure inhomogeneity make the stability and reproducibility of the laser transmitter problematic in serial production quantities. Thus the challenging practical task for miniature low-cost laser transmitter for large-range and highprecision optical radars can be specified as optical pulse duration around $1 \mathrm{~ns}$ with as high as possible peak power (aiming at 100W). For the current driver this means current pulses of several dozen amperes with the pulse duration around $1 \mathrm{~ns}$. Obligatory condition are miniature assembly, simplest construction, and low-cost components.

The goal of this work is to analyze main problems to be solved for development miniature low-cost, high-power nanosecond transmitter optimized for long-distance decimeter-precision lidar.

\section{Driving circuit and a role of each component}

It is possible to show that for a given electrical switch the simplest circuit shown in Figure 1 provides maximum in amplitude and shortest in duration current pulse, which can be used also in the transmitter when replacing the load resistor by a 
laser diode.

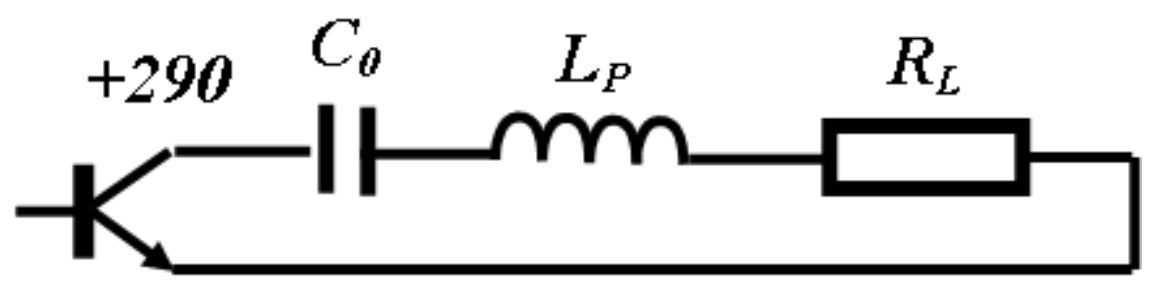

Figure 1. Simplest, most effective and miniature current driver to be realized in miniature assembly. The switch is presented by a bipolar junction transistor (BJT) operating in so called "second breakdown" avalanche mode; capacitor $\mathrm{C}_{0}$ accumulates the energy between the pulses (charges) and discharges across the switch across the load resistor $\mathrm{R}_{\mathrm{L}}$ and entire parasitic inductance of the circuit loop $\mathrm{L}_{\mathrm{P}}$. $\mathrm{L}_{\mathrm{P}}$ limits the current ramp and should be diminished as well as $\mathrm{R}_{\mathrm{L}}$, which is needed only for measuring the current waveform (in our case $\mathrm{R}_{\mathrm{L}}=1 \Omega$ ). In case of optical transmitter the load resistor is replaced with the laser diode.

Very useful for simple but very instructive estimates are the following relations for peak current amplitude $I_{m}$ and FWHM of the current pulse $t_{w}$ valid for an "ideal" switch with infinitely short switching time between the levels $\mathrm{U}_{0}$ (maximum voltage across the switch) and $U_{R}$ (residual voltage across the switch):

$$
t_{w}=2.2 \times \sqrt{L_{P} \times C_{0}} \quad \text { (1) and } I_{m}=\frac{U_{0}-U_{R}}{\sqrt{\frac{L_{P}}{C_{0}}}+R_{L}}
$$

Given the switch is ideal $\left(\mathrm{U}_{\mathrm{R}}=0\right.$, switching time is infinitely short) and load resistor is replaced with a laser diode with zero impedance we obtain the following "quality criterion" for our transmitter: $I_{m} / t_{w}=\frac{U_{0}}{2.2 \times L_{P}}$. It is obvious that for a long-range high-precision radar the quality criterion $\mathrm{I}_{\mathrm{m}} / \mathrm{t}_{\mathrm{w}}$ has to be as large as possible, and thus preferable is highest possible biasing $\mathrm{U}_{0}$ and lowest possible inductance. The biasing voltage is determined by the compromise between mentioned criterion, switching speed of the particular transistor and the maximum voltage that a particular user allows in his system. The parasitic inductance $\mathrm{L}_{\mathrm{P}}$ should always be diminished, and its fundamental limit is determined by the size of the components of circuit shown in Figure 1. (It is obvious that the assembly has to be realized as a 3-D construction of minimal size, and that all components in the construction have to be represented as semiconductor chips).

\section{Results and discussions}

Among commercial avalanche transistors optimal in our case is apparently FMMT415 (ZETEX Semiconductors Inc.) as it combines fairly high biasing 
$\left(\mathrm{U}_{0} \sim 300 \mathrm{~V}\right)$ with reasonably short switching time $(\sim 2 \mathrm{~ns})$. Shown in Figure 2 are our measurement results of the voltage and current waveforms across this switch at different $\mathrm{C}_{0}$ values and relatively large parasitic inductance of $5 \mathrm{nH}$, which cannot be reduced when multilayer ceramic capacitors NP0 type (well operating in nanosecond pulses) have been used with surface mounted transistor package, (but not a chip transistor).

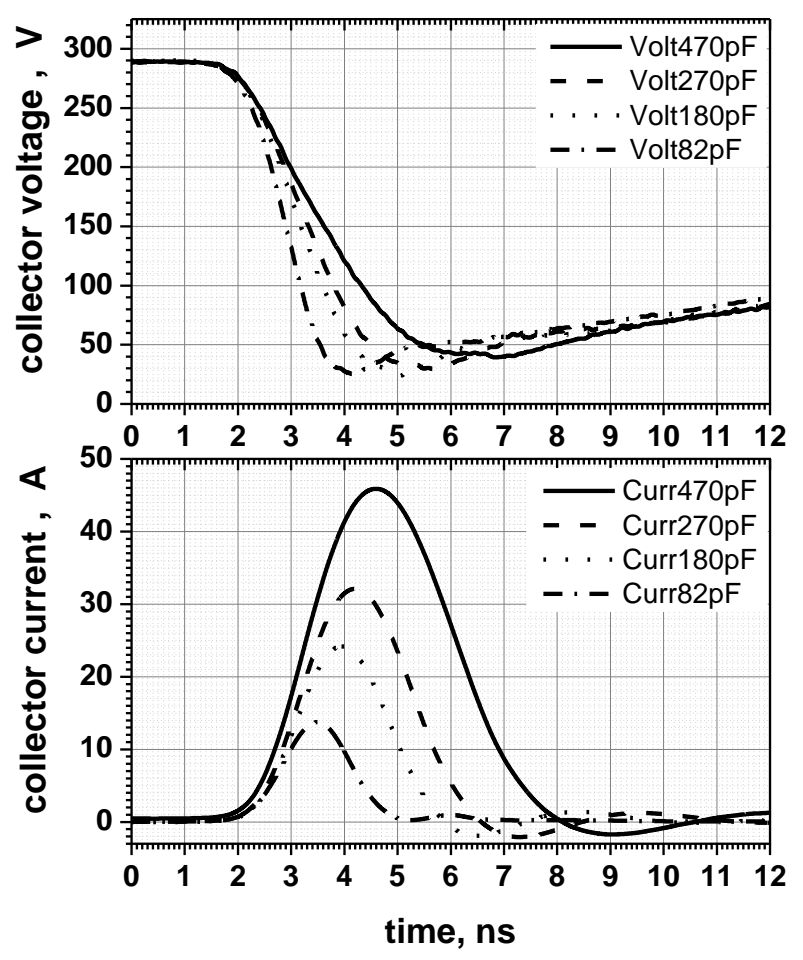

Figure 2. Voltage and current waveforms measured during the switching transient of commercial avalanche transistor FMMT415 (Zetex semicond. Inc.) switched in the circuit shown in Figure 1 with different multilayer ceramic capacitors of NP0 dielectric type, total inductance of the circuit of $5 \mathrm{nH}$ and load resistor $1 \Omega$. Shown current pulses are recovered from the measured voltage waveforms across the load by a numerical procedure on account of $0.8 \mathrm{nH}$ parasitic inductance of the load resistor.

Most instructive for analyzing ways of reduction in the current pulse duration and increase in the pulse amplitude is the presentation of the experimental results in the graph shown in Figure 3. Comparison of the experimental points with the predictions given by simplest relations (1) and (2) allows to arrive to important practical recommendations. 


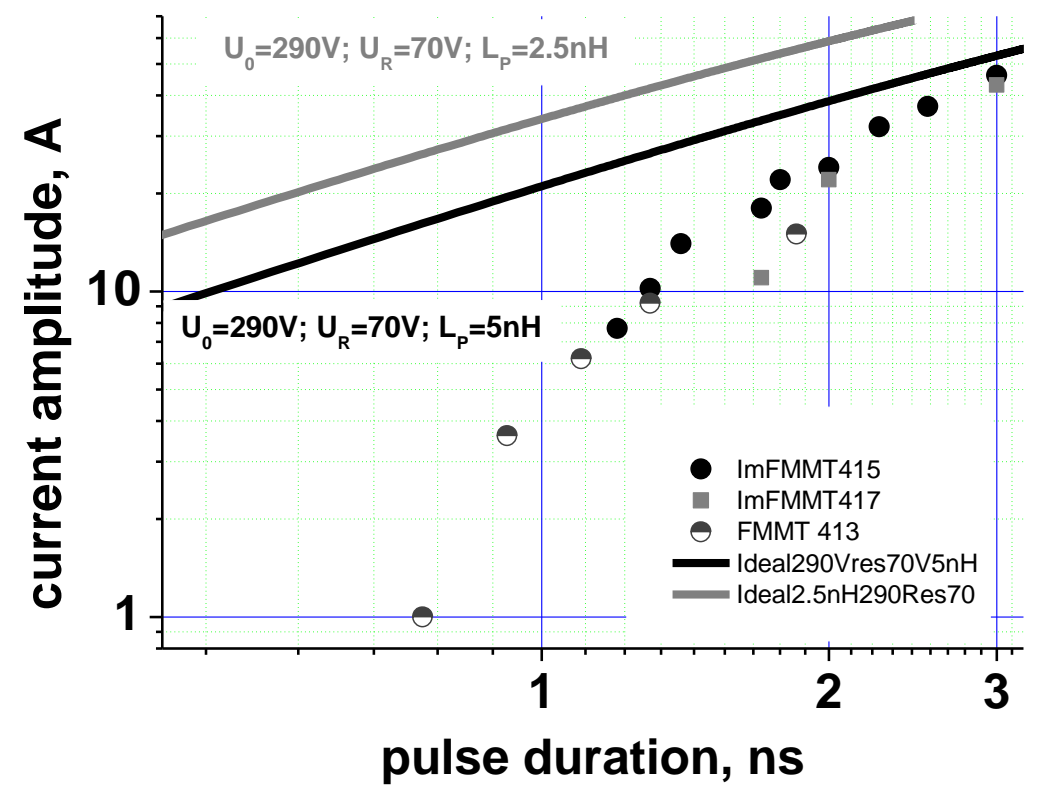

Figure 3. Dependence of peak current on the pulse duration measured using multilayer ceramic NP0 capacitors of different value, one total parasitic inductance of the circuit $5 \mathrm{nH}$, and different commercially available avalanche transistors FMMT 415, 413 and 417 (Zetex Semiconductors). Black and gray solid curves present calculations using formulas (1) and (2). The value of residual voltage $U_{R}=70 \mathrm{~V}$ used in the calculations is taken from the voltage curves in Figure 2 in a timing point corresponding to the peak in the corresponding current waveform. Black curve has to be compared to the experimental points measured for the transistor FMMT415, while the gray curve provides expectations for a switch with infinitely short switching time with $70 \mathrm{~V}$ residual voltage but the inductance reduced down to $2.5 \mathrm{nH}$.

This very rough, but descriptive approach gives good ideas of the main bottle-necks in achieving shorter pulses with increased amplitude. In some cases it require first of all a reduction in the total parasitic inductance by assembling means, in other case main limitation origins from the switching speed of avalanche transistor, and in third case most effective way of increasing $\mathrm{I}_{\mathrm{m}} / \mathrm{t}_{\mathrm{w}}$ ratio is using specially developed surfacemounted capacitors, which will not only reduce parasitic inductance in the assembly, but will provide releasing the charge accumulated in the dielectric in sub-nanosecond range at large-signal mode. (According to our experience the dielectric losses tangent measured in small-signal mode may contradict the results obtained in high-current nanosecond pulse measurements, and optimization of the dielectric require additional labor-consuming tests.) The third factor will be considered elsewhere, while here we concentrate on first two factors. 
One can see from Figure 3 that the best of all available from Zetex avalanche transistors from point of view of maximizing quality criterion $I_{m} / t_{w}$ is FMMT415. Then, for an ideal switch (infinitely short switching time) the calculated for $L_{P}=5 \mathrm{nH}$ curve is presented by black solid line. It gives a bit higher $\mathrm{I}_{\mathrm{m}} / \mathrm{t}_{\mathrm{w}}$ values, but comparable with the experiment for FMMT415 and large $C_{0}$ values (pulse duration $t_{w}$ exceeding 2-3 ns). This means that the switching time of the transistor is comparable with $2 \mathrm{~ns}$, and the current pulse amplitude and duration estimated using formulas (1) and (2) are not far from the experiment, while for shorter pulse durations the difference increases drastically.

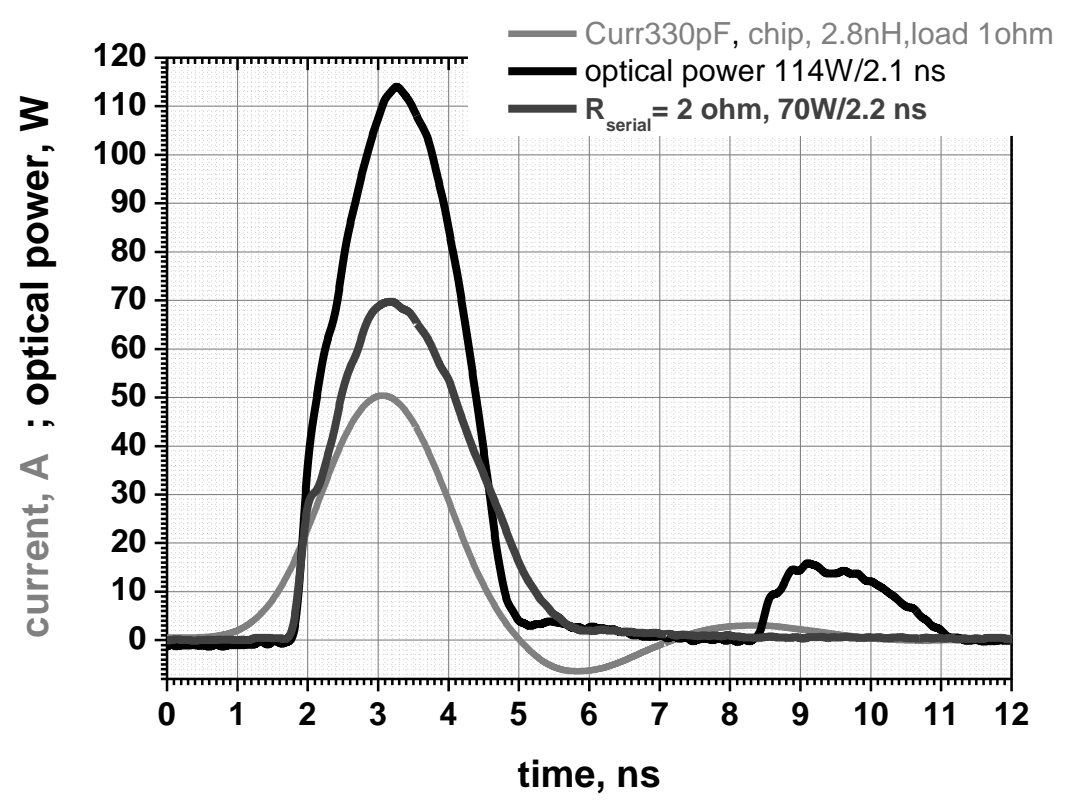

Figure 4. Pumping current pulse using chip of FMMT415 transistor, NP0 capacitor $330 \mathrm{pF} 1 \Omega$ load resistor for current measurement and laser diode; optical response or $114 \mathrm{~W} / 2.1 \mathrm{~ns}$ in presence, however, of a second pulse caused by a current relaxation oscillation. The oscillation is dumped using additionally a serial resistor of $2 \Omega$, which has allowed the second optical pulse to be suppressed, but this reduced the peak power down to $70 \mathrm{~W}$.

We can conclude from Figure 3 that (i) when current pulses longer than 2 ns are required, commercial avalanche transistor FMMT415 is suitable, and current amplitude can be efficiently increased by reduction in the parasitic inductance: for example if inductance were reduced from $5 \mathrm{nH}$ to $2.5 \mathrm{nH}$, the pulse amplitude can be 
increased by a factor of 1.5 - 2 (see gray curve in Figure 3); (ii) when current pulse below $2 \mathrm{~ns}$ in duration were required, no significant win can be obtained from reduction in the inductance, but special avalanche switch has to be designed with shorter switching time, and only then reduction in the inductance value can provide significant win in the current. Actually we have clear vision of how higher-speed avalanche transistors can be designed even using standard Si technology, and the work is already underway. If a current (optical) pulse of $1 \mathrm{~ns}$ in duration is needed, even FMMT415 or 413 can be used, but the current pulse will not exceed 4-5A, and the optical power even with advanced laser diode will be around $10 \mathrm{~W}$ at best. Thus significant increment in optical power for $1 \mathrm{~ns}$ pulses requires simultaneously development of faster avalanche transistor, low-inductance assembly of properly configured components, and, as will be seen from another paper, proper development of the capacitor. Optimistically we may expect an increase in the peak current up to $\sim 20 \mathrm{~A}$, which may provide with an appropriate laser diode peak power of $\sim 40 \mathrm{~W}$.

Shown in Figure 4 is optical pulse obtained with a driver based on FMMT415 transistor and NP0 capacitor. The emitter is a broad-stripe $(100 \mu \mathrm{m})$ three active layer near-infrared $(900 \mathrm{~nm})$ laser diode with efficiency of $2.3 \mathrm{~W} / \mathrm{A}$. After pulsing was observed in optical response due to relaxation current oscillation, which was successfully suppressed by a serial resistor of $2 \Omega$, but the peak power of optical pulse in this case reduced from $114 \mathrm{~W}$ down to $70 \mathrm{~W}$. More technologically advanced way would be reducing the parasitic inductance still further below $2.8 \mathrm{nH}$, which apparently will dump the relaxation oscillation below lasing threshold. This requires, however, replacing NP0 capacitor by specially designed surface-mounted capacitor, which is a subject of another work [13].

\section{Conclusion}

Commercial avalanche transistors allow current/optical pulse generation up to $2 \mathrm{~ns}$ in duration, while at shorter pulses the efficiency of the switch reduces drastically, and special development of optimized $\mathrm{Si}$ avalanche transistor for ns/sub-ns range is needed. Using commercial avalanche transistor chip allows a peak power in a single optical pulse of $70 \mathrm{~W}$ to be achieved with pulse duration down to 2 ns. Further improvement in the peak power and very challenging reduction in the pulse duration down to $1 \mathrm{~ns}$ requires both development of new transistor type and new capacitors, which should also reduce the parasitic inductance of the assembly down to $\sim 1 \mathrm{nH}$.

Suggested here approach should have high potential for large-volume civil market. Indeed, military optical radars that require larger time-of-flight (TOF) ranging, better precision and shorter measurement time loose to our approach drastically in compactness and price that are issues of principal importance, for example, for TOF distance measurements in Civil Engineering when large-size objects such as Hypermarkets, stadiums, airports, etc. are constructed. Moreover, low-cost compact systems utilizing suggested transmitter may create new largevolume markets such as radar utilization in navigation systems for small boats, launches and yachts. 


\section{Acknowledgements}

This work was performed under the government order of the Ministry of Education and Science of RF. M.C. Wurz. and A. Filimonov would like to thank the DAAD Grant "Scientific Partnership with St. Petersburg State Polytechnic University and Leibniz Universität Hannover”.

\section{References}

1. A. Biernat and G. Kompa "Powerful picosecond laser pulses enabling highresolution pulsed laser radar", J. Optics, V. 29, pp.225-228, 1998.

2. A. Kilpela and J. Kostamovaara, "Laser pulser for a time-of-flight laser radar", Rev. Sci. Instr., V.68, pp. 2253-2258, 1997.

3. R. Myllyla“, J. Marszalec, J. Kostamovaara, A. Ma“ntyniemi, and G.-J. Ulbrich, "Imaging distance measurements using TOF lidar", J. Opt. 29, 188-193, 1998

4. J. Li, X. Zhong, J. Li, Z. Liang, W. Chen, Z. Li, and T. Li, "Theoretical Analysis and Experimental Study on an Avalanche Transistor-Based Marx Generator", IEEE Trans. Plasma Science, vol. 43, no. 10, pp. 3399-3405, Oct. 2015, doi: 10.1109/TPS.2015.2436373.

5. G. Duan, S.N. Vainshtein, J. T. Kostamovaara, "Modified high-power nanosecond Marx generator prevents destructive current filamentation" in press IEEE Trans. on Power Electronics: Manuscript ID TPEL-Reg-2016-10-2004.

6. S. N. Vainshtein, G. Duan, A. V. Filimonov, and J. T. Kostamovaara, "Switching Mechanisms Triggered by a Collector Voltage Ramp in Avalanche Transistors With Short-Connected Base and Emitter", IEEE Trans. Electron Devices, vol. 63, no. 8, pp. 3044-3048, Aug. 2016. doi: 10.1109/TED.2016.2581320.

7. W.B. Herden, "Application of avalanche transistors to circuits with a long mean time to failure", IEEE Trans. Instrum. Meas., V. IM-25, pp. 152-160, 1976.

8. B.Q. Streetman, Solid State Electronic Devices. Englewood Cliffs, Inc, N.J., pp. 344-346, 1972.K.

9. S.N. Vainshtein, G.S. Simin, and J.T. Kostamovaara, "Deriving of single intensive picosecond optical pulses from a high-power gain-switched laser diode by spectral filtering", Journal of Applied Physics, V.84, No. 8, pp. 4109-4113, 1998.

10. S.N. Vainshtein and J.T. Kostamovaara, "Spectral filtering for time isolation of intensive picosecond optical pulses from a Q-switched laser diode", Journal of Applied Physics, V.84, No. 4, pp. 1843-1847, 1998.

11. S.N. Vainshtein, J.T. Kostamovaara, R.A. Myllyla, A.J. Kilpela, K.E.A. Maatta " Automatic switching synchronization of serial and parallel avalanche transistor connections", Electron. Lett., V. 32, N 11, pp. 950 - 952, 1996.

12. J. Lee, Y.J. Kim, K. Lee, S. Lee, S.W. Kim, "Time-of-flight measurement with femtosecond light pulses" Nature Photonics V. 4, pp.716-720, 2010

13. A. Filimonov, S. Vainshtein, V.E. Zemlyakov, V.I. Egorkin Investigation of electro-physical and transient parameters of energy accumulating capacitors applied in nanosecond and sub-nanosecond high-current avalanche switches. Lecture Notes in Computer Science. 2016. Vol. 9870. P. 731-737 